\title{
TOWARDS DIRECT DETECTION OF GOLD BEARING ROCK FORMATIONS FROM SEISMIC DATA, ST. IVES GOLD CAMP, WESTERN AUSTRALIA
}

\author{
Christopher Harrison ${ }^{1}$ And Milovan Urosevic ${ }^{2}$ Curtin University Of Technology \\ C.Harrison1@Postgrad.Curtin.Edu.Au ${ }^{1}$,Milo@Geophy.Curtin.Edu.Au ${ }^{2}$
}

Key Words: Mining, Seismic, Inversion, Attributes,

\section{INTRODUCTION}

In 2004, Gold Fields Limited and Curtin University of Technology undertook a project to assess the feasibility of the application of high resolution reflection seismic for gold exploration in Western Australia. A large scale regional survey several years before had indicated that deeper structures can be successfully imaged with course seismic acquisition techniques. These surveys provided images on a regional and campscale only while shallower structures of direct interest to exploration remained unresolved. The acquisition of high resolution seismic intended to detect economically viable targets for mineral exploration, turned out to be not a trivial endeavour. Seismic lines which traversed over and along busy mine site roads, around restricted areas, and through irregular, jagged terrain result in crooked-line geometry, often saturated with ambient noise, and running in an unfavourable direction with respect to the dominant trend of the major structures. This creates out-of-plane reflections and limits the effectiveness of pre-stack and post- stack imaging techniques and severely affects the calibration with sonic logs. While mine-sites generally have an abundance of borehole drilling data, sonic logs are in sparse supply and often restricted to shallow depths by hydrocarbon standards (200-900 meters), which presents a difficulty for seismic data inversion and subsequent lithological interpretation.

\section{AREA OF INVESTIGATION}

This study focuses on the East Victory seismic line at the St. Ives gold camp in Western Australia. The St. Ives gold camp is located on the Yilgarn craton, an archaean mafic sequence made up of basalts, ultra-mafics and interflow sediments. These formations are found primarily in a southerly plunging anticline, overlain with unknown crusts of felsic composition intruded by dolerites and felsic porphyries (Drummond et al, 2000). As illustrated in Error! Reference source not found., the East Victory seismic line was orientated approximately in the dip direction of the dominant anticline in the area, with several other major features including the Boulder-Lafroy fault and the Foster thrust intersecting with the seismic line.

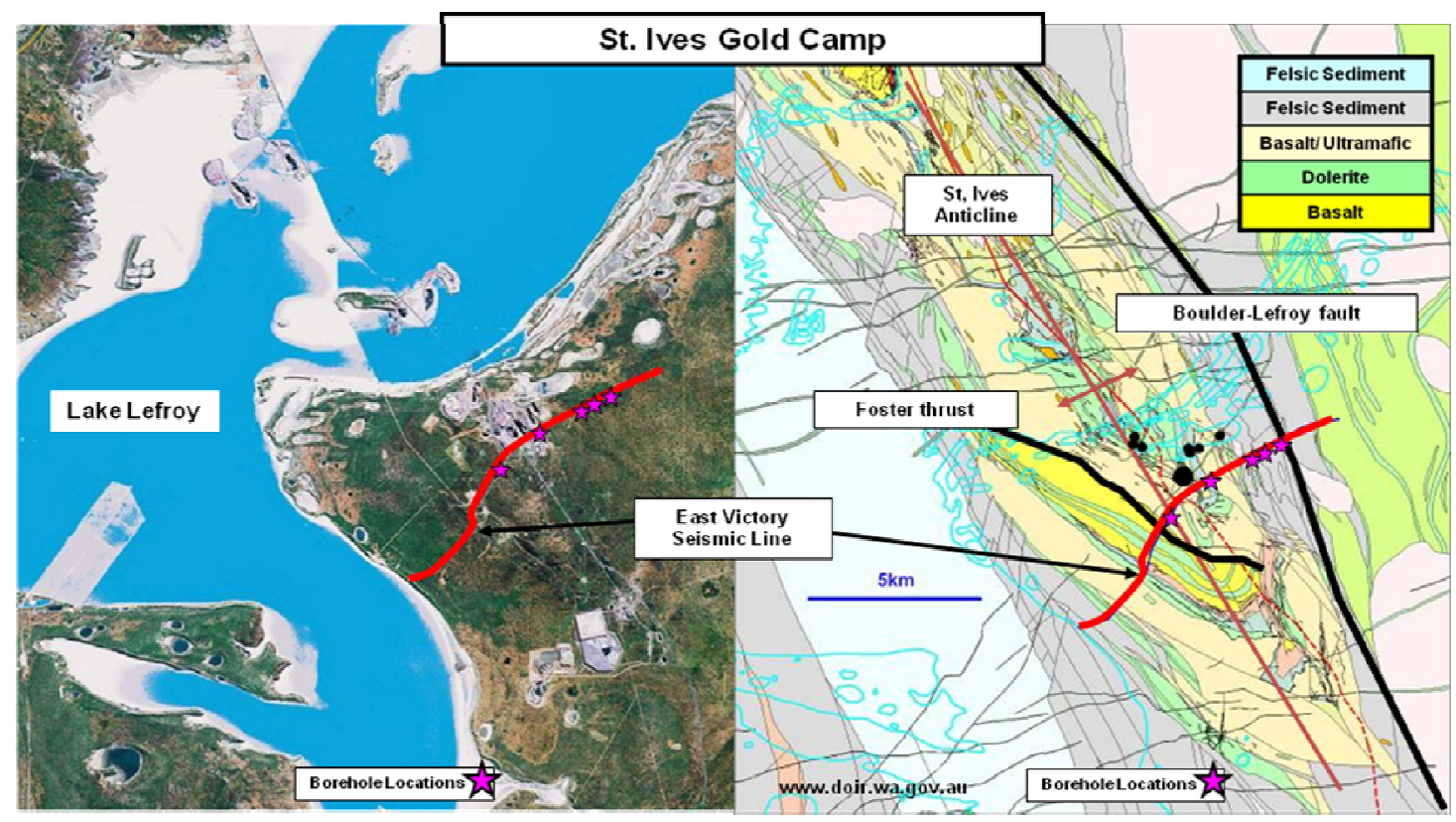

Figure 1. Map view and geological interpretation view of the East Victory seismic line. 


\section{SEISMIC DATA PROCESSING}

To maximize predictive results, processing of seismic data in hard-rock environments has to take into consideration the end objective of inversion and attribute analysis. The complex structures, highly heterogeneous regolith zone, high ambient noise, and variable source-receiver couplings, however, present serious challenges to preservation of relative amplitudes of seismic data. Given that, inversion and attribute analysis require minimizing or eliminating distortions in the original reflection amplitude, frequency, and phase must be overcome for seismic imaging to be of value for mineral prediction.

One of the key requirements for accurate seismic imaging is accurate velocity analysis which, in hard-rock environments, could only be achieved by the application of the constant velocity stack analysis in combination with dip moveout corrections and/or common surface reflection (CSR) approach. 2D line orientated in the dip direction of the dominant structures maximizes pre-stack and post-stack migration techniques, thus generating interpretable structural images. Controlling both the image quality for structure interpretations and limiting image distortions will ensure less ambiguous results when calibrating borehole sonic log information to the seismic image.

This study utilized a conventional processing flow ending with a post-stack explicit finite difference time migration. Multiple iterations of constant velocity stack combined with an interactive semblance based velocity analysis scheme and dip move-out corrections were needed to arrive at an accurate velocity model. Band-pass filters were designed to retain the original reflection frequencies of approximately $30-90 \mathrm{~Hz}$. Random noise, which generally arises from constant vehicle movement and mine operations, was attenuated by stacking and post-stack application of F-X deconvolution. Pre-stack migration has been suggested as more accurate for imaging of shallow targets (0-500 meters) by Pasasa et al (1998) due to its ability to resolve images with low signal-to-noise ratio. In a complex hard-rock environment pre-stack imaging does not readily leads itself to a high quality image. In fact as shown below, post-stack imaging on the East Victory line was highly effective due to the wealth of data available for reflection verification.

The resulting post-stack image for the East Victory line are shown in Figure 2. The inset map view shows the borehole positions as well as significant camp-scale features. Reflections were confirmed through projections of these features including, the Foster Thrust, the Repulse fault, and the Condensor dolerite. The St. Ives anticline structure is seen quite clearly on this image. The resultant images were zero phased prior to inversion and attribute analysis. The accuracy of seismic inversion and attribute analysis is particularly important for the shallow structures that are of immediate exploration interest $(0-500 \mathrm{~m})$. The blue box illustrated on Figure 2 shows where borehole data was located allowing for seismic inversion and attribute analysis of the East Victory seismic line. 


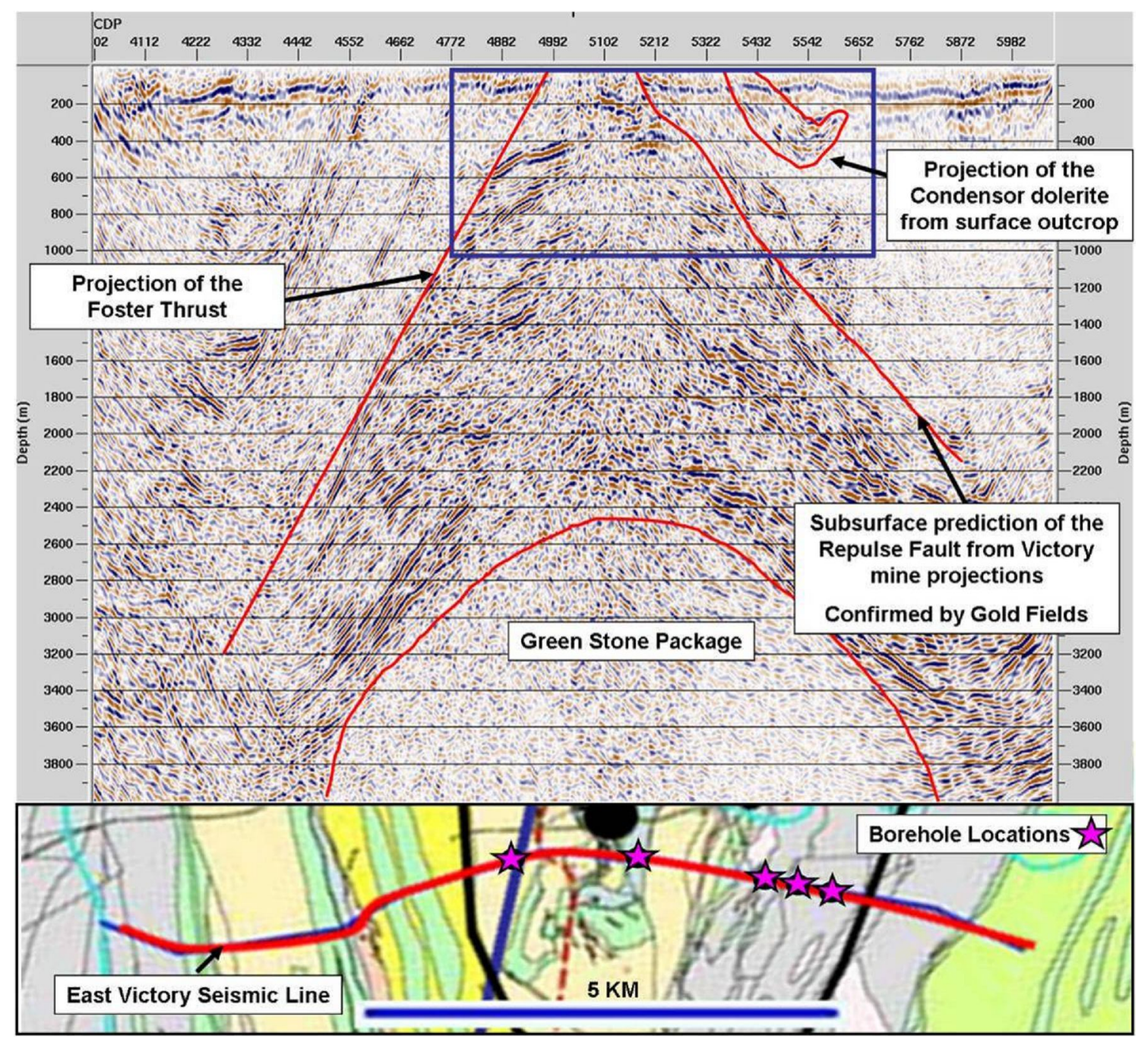

Figure 2. The post-stack migrated East Victory seismic line.

\section{SEIMSIC TO WELL TIE}

A trade-off always exists between what structure is visually extractable from a seismic image and what is statistically useful for predicting rock properties from the seismic data. Predicting rock properties requires calibration of sonic $\log$ with the seismic images. Synthetic seismograms, generated from the sonic data matched with the seismic image give a statistical basis for the identification of lithology, structural features, and alteration resulting in possible prediction of gold hearing formations. However, calibration of the seismic image with the sonic data is a non-trivial process for several reasons. One being often erroneous sonic data acquired in hard-rock environments, the other is contained in the inability of 2D seismic data to accurately represent largely 3D complex structures in this environment. In some cases the sparseness of reflection events do not really fit to the convolution model of seismic trace.

Good quality full-waveform sonic (FWS) data are essential but rarely available from hard-rock environments. With a low signal-to-noise ratio, processing algorithms will distort the original reflection signal making sonic / seismic calibrations imperfect. Consequently, the calibration process as well as seismic inversion and attribute analysis tend to be poorly constrained thus necessitating corroborative on-site geological information.

As illustrated in Figure 1 and Figure 2, five boreholes with sonic logs were available for calibration on the East Victory seismic. To first verify if statistical information could successfully be extracted from the sonic data, rock characterization was carried out on all boreholes utilizing $\mathrm{Vp} / \mathrm{Vs}$ ratio, acoustic impedance, and elastic impedance. Hydrocarbon exploration has used these rock characteristics to make predictions on 
porosity, fluid content, and lithology in seismic images. Analogous responses in hard-rock environments would be mineralization, shear zones, and lithology. Extant rocks were further cross-plotted based on rock type, structure type, and alteration type.

With reliable rock characterization, reflections of the synthetic seismograms and seismic data were matched, utilizing multiple iterations of wavelet extraction and matching for correlation of the two data sets. Figure 3 shows the sonic data calibrated with the seismic data.

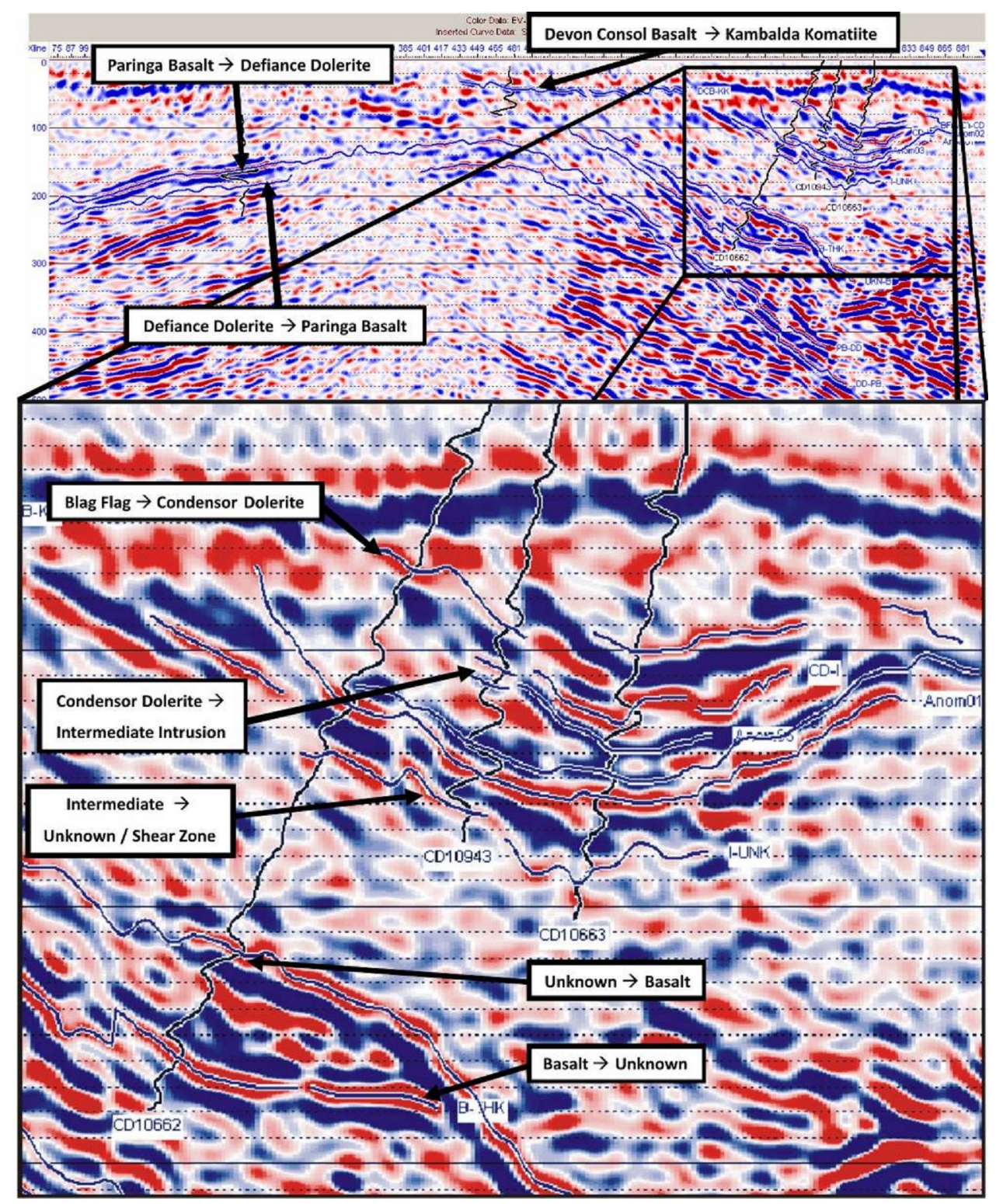

Figure 3. Synthetic seismograms correlated with seismic data. Main contacts have been traced and labelled.

\section{AMPLITUDE VERSUS OFFSET}

Changes in velocity, density, and Poisson's ratio in rock characteristics allow for what is known as amplitude versus offset (AVO) studies. These techniques are a relatively untested technology in mineral exploration in Western Australia but have shown great promise for mineral content and structure predictions. Figure 4 shows AVO effects observed on sonic data through CD10663. AVO effects, albeit weak, can be seen on the zero to 1000 meter offset range at both the high gold content and rock change zones. Calculations reveal the reflection coefficient magnitude to be below 0.06 with no polarity changes. 


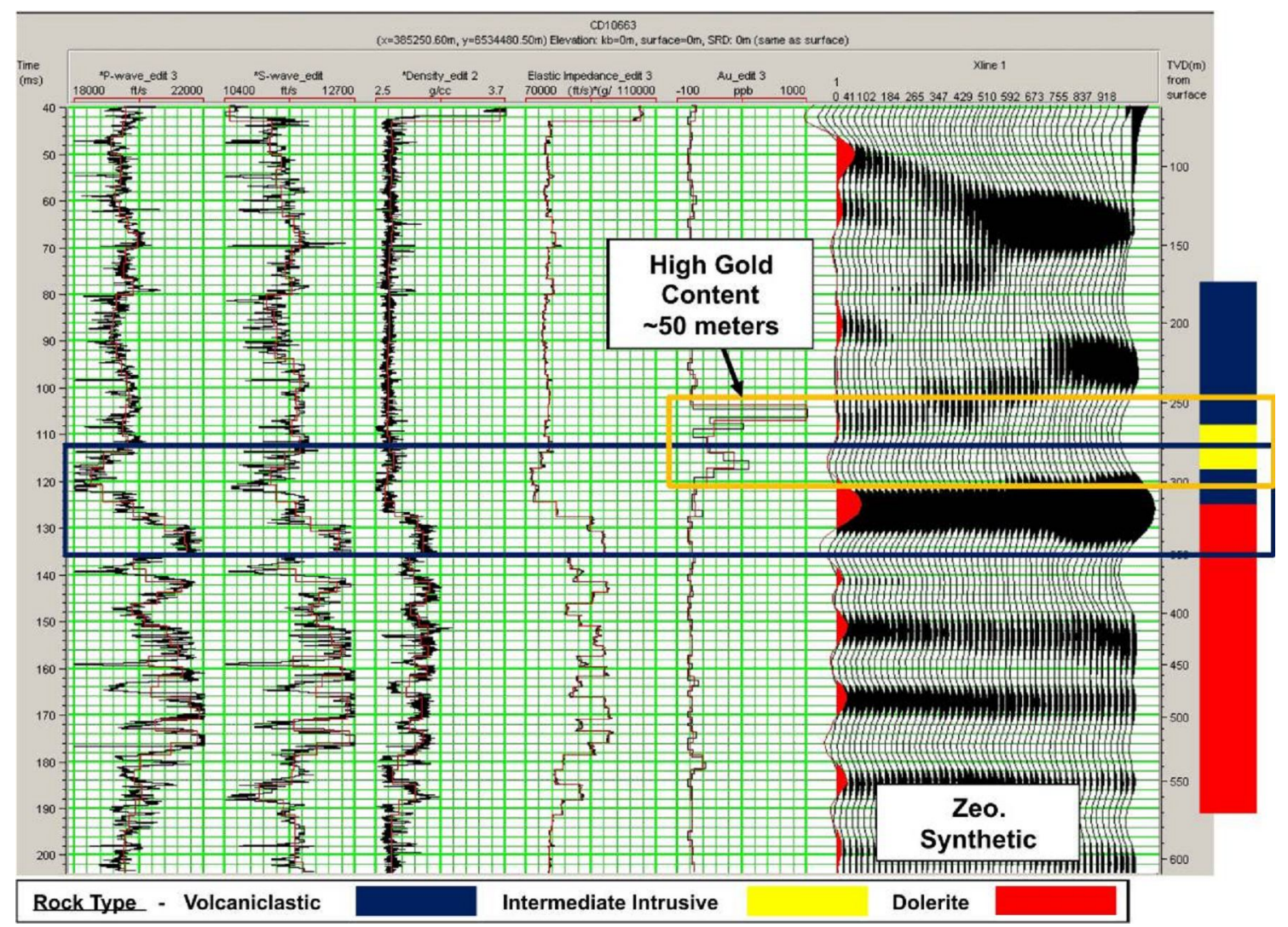

Figure 4. Amplitude versus offset calculations for borehole CD10663. High gold content zone is coincident with an intermediate intrusive through a volcaniclastic

\section{SEISMIC INVERSION}

Consistent inversion results for lithology predictions are difficult to achieve in hard-rock environments. Low signal-to-noise ratio, lack of check-shots, wavelet distortions, sonic logging errors, and typically poor correlation between synthetic seismogram derived from sonic log and seismic data makes inherently nonunique impedance predictions process even more challenging. Reduction of inversion error can be achieved through utilization of a large number of sonic logs for validation of results. However, large volumes of sonic data are not available in hard-rock environments at present.

Figure displays where sonic to seismic correlations can be considered reliable. The enlarged location revealed strong synthetic seismogram as well as observed AVO effects correlations with seismic reflections. With no check shot, however, multiple iterations of log stretching and time-and-phase shifting to achieve an average 0.723 cross-correlation coefficient was required. With these high correlation values, multiple inversions runs were conducted to find and optimum approach to inversion in hard-rocks.

The results of the application of coloured and sparse-spike maximum likelihood inversion schemes are shown in Figure 5. The coloured inversion results appear smoother with high correlations with intermediate intrusive zones near the Condensor doleriate. The sparse-spike results appear, strangely enough, spiky with better visual separation between dolerites and softer surrounding rock. High gold content locations are highlighted and observed throughout intermediate rock contacts. 

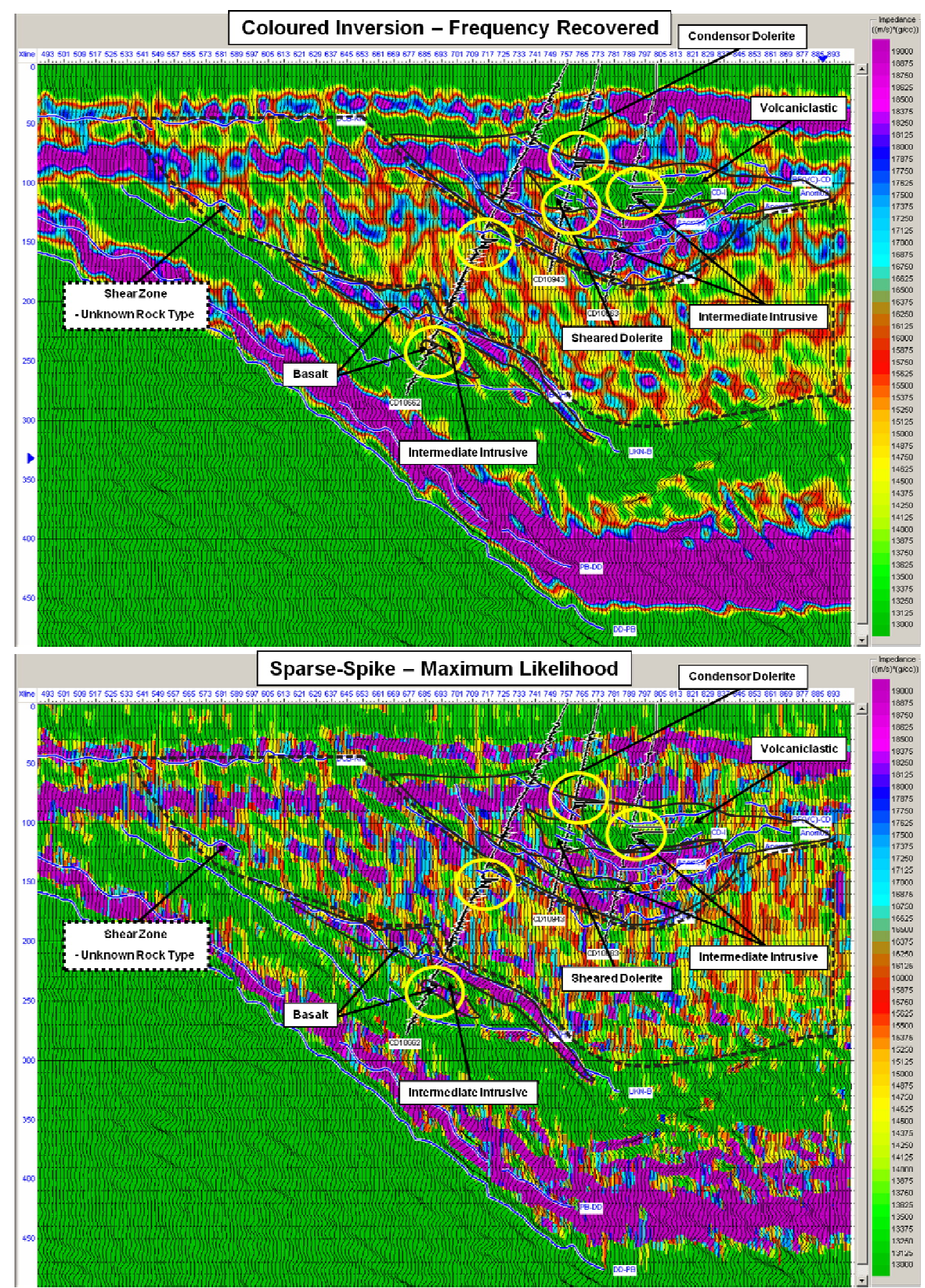

Figure 5. Coloured inversion with recovered frequency content (top) and sparse-spike inversion (bottom) of the East Victory Seismic line.

\section{ATTRIBUTE ANALYSIS}

Seismic attribute analysis was also conducted to predict lithology and gold content on the East Victory seismic line. For predictions throughout the seismic section, attribute analysis utilizes suspected ties between known rock properties from the log and wave-field attributes. Validating predictions from attribute analysis requires a greater number of borehole sonic logs than seismic inversion analysis required. Given the absence of a crucial volume of good quality sonic logs, for corroboration, attribute results remain ambiguous.

Hydrocarbon exploration has utilized attribute analysis studies for reservoir characterization, lithology prediction, and porosity forecasting. Analogous properties in hard-rock environments might include alteration plume prediction, gold content forecasting and lithology prediction. The gold content was the only quantifiable mineralization log-property available for gold mineralization prediction analysis. Two types of 
analysis were completed using geostatistical analysis for gold prediction on the East Victory line. The first was an operator of length 1 with the highest correlating attribute, dominant frequency, and the second was an operator of length 7 with 5 attributes; integrated absolute amplitude, narrow filter range 25/30 - 35/40, integrate, instantaneous phase, and narrow filter range 45/50-55/60. High gold content predictions in red can be seen on Figure 6.

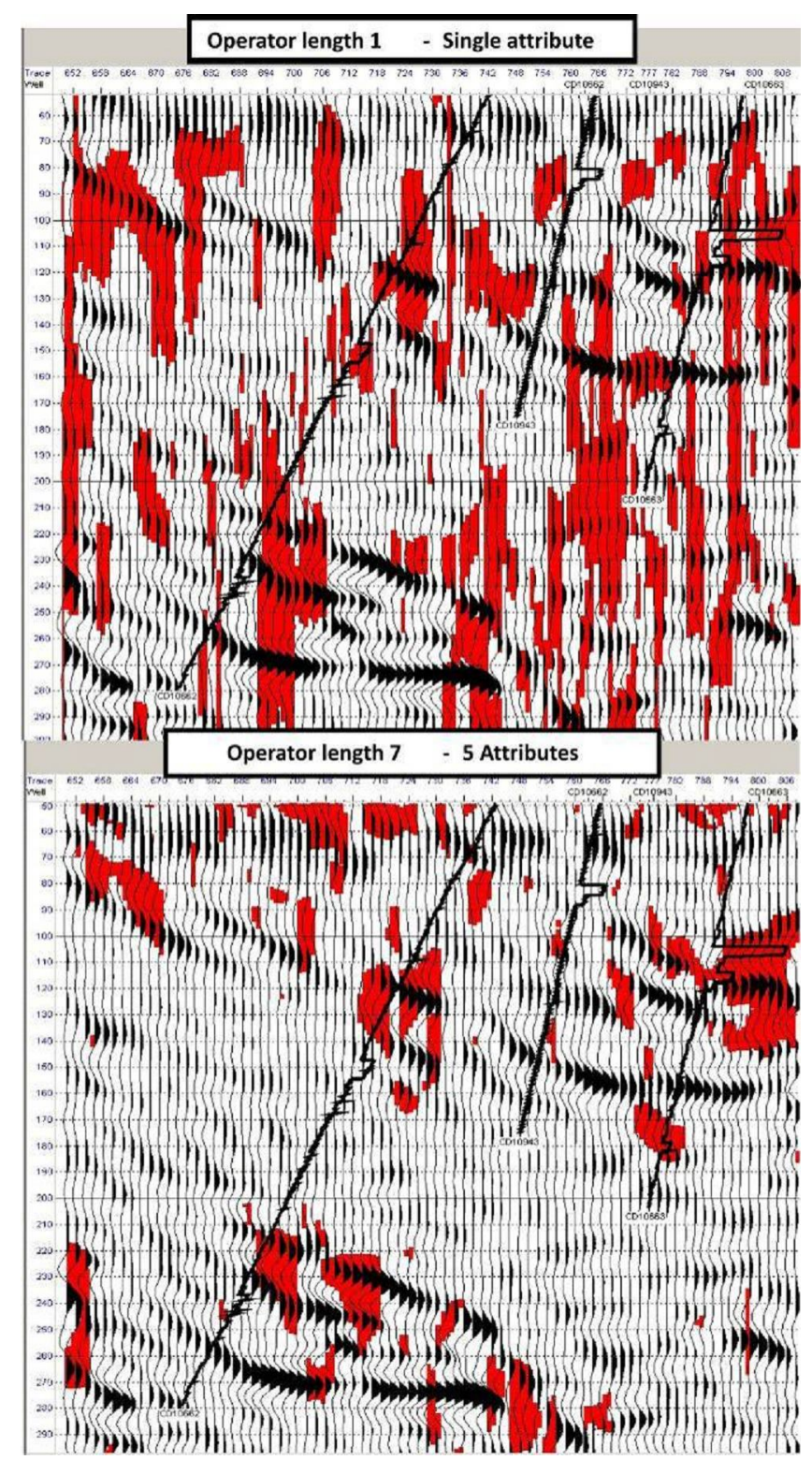

Figure 6. Gold content predictions through attribute analysis on the East Victory seismic line. Red indicates zones of possible high gold content ( $+4000 \mathrm{ppb})$

\section{CONCLUSION}

Processing of the East Victory seismic line yielded accurate seismic image and identification of the main rock units that was largely helped by an abundance of surface geological interpretations and availability of good quality borehole data. Seismic inversion further shows promise for rock-type prediction, and identification of gold bearing structures away from boreholes. However, given the lack of sonic log data for cross-checking of results, inversion errors remain poorly accessed. Seismic attribute analysis for gold zone predictions also showed promise, but the value of the results remains ambiguous due to the lack of borehole data for cross checking of the results. Further advances in this area and investigations into seismic based mineral predictions will require a larger number of sonic data and 3D seismic images. 


\section{ACKNOWLEDGEMENT}

This research is sponsored by Centre of Excellence for High Definition Geophysics at the Department Of Exploration Geophysics, Curtin University. We also acknowledge continuous software support of Landmark Graphics (A Halliburton Company) and Hampson \& Russell software services (CGGVeritas).

\section{REFERENCES}

Drummond, B. J., Goleby, B. R., Own, A. J., Yeates, A. N., Swager, C., Zhang, Y., and Jackson, J. K., (2000). "Seismic reflection imaging of mineral systems: Three case histories:." Geophysics 65: 1852- 1861.

Pasasa, L., Wenzel, Friedemann, and Zhao, Ping, (1998). "Prestack Kirchhoff depth migration of shallow seismic data." Geophysics 63(4): 1241-1247. 